\title{
« Des pilules contre tous les microbes?» Programme d'éducation communautaire pour une utilisation judicieuse des antibiotiques
}

\author{
Carson $\mathrm{M}^{1^{*}}$, Patrick $\mathrm{DM}^{2,3}$ \\ ${ }^{1}$ Organisme de santé publique Alberta Health Services, Edmonton (Alberta) \\ ${ }^{2}$ École de santé publique et de santé des populations, Université de la Colombie-Britannique, Vancouver (Colombie- \\ Britannique) \\ ${ }^{3}$ Centre de contrôle des maladies de la Colombie-Britannique, Vancouver (Colombie-Britannique)
}

*Correspondance : mary.carson@albertahealthservices.ca

\section{Résumé}

Le programme " Des pilules contre tous les microbes? 》 est un programme éducatif communautaire et professionnel qui vise à s'attaquer à la résistance aux antibiotiques en réduisant l'utilisation inappropriée de ces médicaments. Des documents ont été préparés à l'intention des médecins, des pharmaciens, des infirmières et du public, y compris les enfants, leurs parents et personnes soignantes, les enseignants, les employeurs et les travailleurs, et les établissements de soins de longue durée. Le programme s'appuie sur quatre stratégies clés : un message cohérent ( $p$. ex., se laver les mains est la meilleure manière d'éviter la propagation des infections); le réseautage (par l'intermédiaire de membres de comité dévoués qui ont mobilisé les ministères gouvernementaux, les organisations professionnelles, les organisations de soins de santé, le milieu universitaire, l'industrie et les entreprises, ainsi que les groupes communautaires); l'harmonisation des intérêts (p. ex., demander aux étudiants en soins infirmiers et en médecine d'offrir un programme sur la résistance aux antimicrobiens (RAM) aux élèves de $2^{\mathrm{e}}$ année dans le cadre de leur programme d'études en santé communautaire); et la maîtrise des coûts (p. ex., s'associer à d'autres organisations pour la distribution du matériel et la mise en commun des coûts administratifs et des frais d'impression entre les programmes « Des pilules contre tous les microbes? » de l'Alberta et de la Colombie-Britannique). De nos jours, le milieu scientifique et le monde médical semblent de plus en plus disposés à discuter des risques associés à l'utilisation des antibiotiques et le public a tendance à être de plus en plus conscient que la RAM est liée à une utilisation inappropriée et excessive des antibiotiques.

\section{Introduction}

Les organismes résistants aux antibiotiques sont responsables d'une proportion toujours croissance des infections d'origine communautaire au Canada. Tous les médecins connaissent bien la réalité d'aujourd'hui du Staphylococcus aureus résistant à la méthicilline (SARM) acquis dans la collectivité pour les infections de la peau et des tissus mous, ainsi que la croissance très marquée de la résistance aux fluoroquinolones et au triméthoprime-sulfaméthoxazole des agents pathogènes des voies urinaires acquis dans la collectivité, comme $E$. coli.

Le programme " Des pilules contre tous les microbes? » est un programme éducatif communautaire qui vise à s'attaquer à la résistance aux antibiotiques en réduisant l'utilisation inappropriée de ces médicaments. II a débuté en 1997 comme petit projet pilote de six mois à Grande Prairie, en Alberta; le programme s'est ensuite étendu jusqu'à Edmonton en 2000, et au reste de l'Alberta et à la Colombie-Britannique en 2005. Des documents ont été préparés à l'intention des médecins, des pharmaciens, des infirmières et du public, y compris les enfants, leurs parents et personnes soignantes, les enseignants, les employeurs et les travailleurs, ainsi que les établissements de soins de longue durée. Les programmes sont appuyés par des documents imprimés, un site Web, de la publicité à la télévision et dans les moyens de transport, ainsi qu'un compte Twitter. Le site Web du programme 
«Des pilules contre tous les microbes? » est bilingue, et quelques documents imprimés existent en plusieurs langues (1).

La Colombie-Britannique a connu des réductions du taux de prescription communautaire à l'échelle de la population, en particulier en ce qui concerne les infections des voies respiratoires chez les enfants, la cible initiale principale du programme (2). En Alberta, la mise en œuvre d'une stratégie de gestion des antimicrobiens qui comprend la formation du personnel et la rétroaction sur les taux de prescription des antibiotiques a permis d'obtenir des réductions systématiques de la prescription des antibiotiques dans les centres de soins de longue durée (données inédites; Mary Carson). Cet article a pour objectif de résumer les principales stratégies de ce programme communautaire de gestion des antimicrobiens.

\section{Quatre stratégies clés}

Le succès du programme "Des pilules contre tous les microbes? » s'explique en partie par ses quatre stratégies : message cohérent, réseautage, harmonisation des intérêts et maîtrise des coûts.

\section{Message cohérent}

Trois messages clés ont été établis au moment de la création du programme et continuent encore aujourd'hui d'être le point central des efforts éducatifs. Ils ont été incorporés aux documents du programme destinés à tous les niveaux, des professionnels de la santé aux enfants d'âge préscolaire.

- $\quad$ Se laver les mains est le meilleur moyen de contrer la transmission d'infections.

- Les virus et les bactéries sont différents et les antibiotiques ne fonctionnent pas contre les virus.

- Utiliser les antibiotiques judicieusement pour limiter l'apparition de la résistance aux antibiotiques.

Même s'il est relativement facile, par exemple, de montrer aux jeunes enfants comment bien se laver les mains, la résistance aux antibiotiques est bien plus difficile à enseigner. Cependant, même les jeunes enfants peuvent découvrir les différents types de microbes, et apprendre que les antibiotiques « entrent » dans les bactéries, mais pas dans les virus. Nous estimons que la cohérence et la répétition de ces messages clés étaient essentielles à la réalisation réussie d'une campagne de sensibilisation du public.

\section{Réseautage}

Le réseautage est une activité essentielle du programme "Des pilules contre tous les microbes? ", car c'est grâce aux partenaires communautaires que le programme a été lancé et qu'il continue d'être exécuté. Une grande partie de ces efforts est réalisée par les structures de comités du programme «Des pilules contre tous les microbes? " en Alberta et en Colombie-Britannique. Au départ, ces comités comprenaient un microbiologiste médical et un spécialiste des maladies infectieuses, un pharmacien spécialisé dans l'utilisation des agents antimicrobiens et dans les maladies infectieuses, un pharmacien provenant de l'industrie pharmaceutique, une infirmière associée au programme de lignes directrices de pratique clinique, un professeur clinicien provenant d'une faculté de pharmacie, et un représentant d'une organisation non gouvernementale du domaine de la santé. Par la suite, des épidémiologistes, des membres des ordres professionnels et des professionnels de la santé travaillant dans le domaine de la santé publique, des soins de longue durée et de la santé au travail se sont joints à l'équipe.

Les membres du comité ont fait preuve d'un dévouement exceptionnel dans l'élaboration du programme " Des pilules contre tous les microbes? " et ont fait don de leur temps sans s'attendre à une rémunération ou à une reconnaissance personnelle. Ils ont également mobilisé un vaste réseau de partenaires communautaires, y compris des ministères gouvernementaux, des organisations de professionnels de la santé, des établissements universitaires, l'industrie et les entreprises, les établissements de soins de longue durée, les écoles, les 
enseignants, les garderies, les groupes confessionnels, les organisations de jeunes et les citoyens, qui ont tous aidé à diffuser le programme.

\section{Harmonisation des intérêts}

Comme le programme « Des pilules contre tous les microbes? " s'appuie dans une large mesure sur les partenariats avec des groupes ou des organisations externes pour la réalisation du programme, il est important de trouver des partenaires dont les intérêts s'harmonisent, puis d'élaborer ou d'adapter des programmes qui répondent à leurs besoins.

On peut citer comme exemple le programme de $2^{e}$ année, conçu pour être utilisé par des présentateurs invités en salle de classe. Le programme "Des pilules contre tous les microbes? " fournit une formation à l'intention des présentateurs, une trousse d'enseignement et des ressources à l'intention des élèves de $2^{\mathrm{e}}$ année et de l'école, alors que le programme est principalement réalisé par des étudiants en soins infirmiers et en médecine dans le cadre de leurs exigences en matière de santé communautaire. Ce modèle présente l'avantage d'éduquer les jeunes enfants et les futurs travailleurs de la santé et fait partie des activités essentielles du programme « Des pilules contre tous les microbes? » depuis 1997.

Le programme de $2^{\mathrm{e}}$ année dépend du maintien de relations de collaboration avec les facultés de soins infirmiers et de médecine et de l'harmonisation des documents du programme " Des pilules contre tous les microbes? " avec les attentes scolaires du collège ou de l'université. Cette approche est utilisée pour la majorité de nos programmes, car elle facilite la dissémination des messages clés tout en offrant un service qui répond aux besoins des organisations partenaires.

\section{Maîtrise des coûts}

Les coûts de la réalisation du programme « Des pilules contre tous les microbes? » à l'échelle provinciale en Alberta s'élèvent à environ 5 cents par Albertain par année. Plusieurs facteurs contribuent à la maîtrise des coûts, y compris la dépendance à l'égard des organisations partenaires pour la réalisation du programme et les économies d'échelle associées à la mise en œuvre des programmes « Des pilules contre tous les microbes? » en Alberta et en Colombie-Britannique. On peut citer comme exemple de dépendance à l'égard des organisations partenaires le fait qu'au lieu d'un publipostage adressé aux entreprises de l'Alberta concernant les documents disponibles pour le lieu de travail, nous nous sommes associés au programme d'immunisation en milieu de travail d'Alberta Health Services et aux Infirmières de l'Ordre de Victoria du Canada pour distribuer des trousses de documents destinés à l'employeur et au travailleur aux entreprises parallèlement aux cliniques d'immunisation contre l'influenza en milieu de travail. En ce qui concerne les économies d'échelle, les programmes « Des pilules contre tous les microbes? » en Alberta et en Colombie-Britannique bénéficient de frais d'impression et de coûts administratifs réduits en raison du regroupement des commandes d'impression et de l'utilisation des mêmes trousses d'enseignement et ressources Web dans les deux provinces.

\section{Discussion}

L'élaboration et la réalisation des programmes ne sont pas choses faciles. La résistance aux antibiotiques est un concept difficile, non seulement du point de vue de l'éducation du public, mais également de celui des professionnels de la santé. Il peut être décourageant de surmonter l'idée que la résistance aux antibiotiques n'est pas un problème urgent, et cela demande de la persévérance et de l'endurance. Cependant, nous avons découvert qu'avec le temps, le public est devenu plus conscient de ce qu'est la résistance aux antibiotiques et de ce qui la cause. En 1998, 27 \% des personnes interrogées dans le cadre d'une enquête publique étaient capables de donner la définition correcte de la résistance aux antibiotiques. Dans le cadre d'une enquête publique menée en 2014 en Colombie-Britannique, 77 \% des personnes interrogées étaient capables d'attribuer la résistance aux antibiotiques à l'utilisation inappropriée ou excessive d'antibiotiques. Les données de plus en plus nombreuses concernant les risques mondiaux et personnels associés à l'utilisation des antibiotiques et le fait que le milieu 
scientifique et le monde médical sont de plus en plus disposés à discuter de ces risques dans les médias sont des facteurs importants de sensibilisation du public.

À la suite de l'excellent travail international accompli dans le domaine de la résistance aux antimicrobiens $(3,4)$, l'Agence de la santé publique du Canada a dirigé l'élaboration du Plan d'action fédéral sur la résistance et le recours aux antimicrobiens (5). II est important de noter que la stratégie canadienne aborde l'utilisation des antibiotiques en agriculture, ainsi que la nécessité de mettre en place un système de suivi de l'utilisation des agents antimicrobiens dans le domaine agroalimentaire (5). Nous sommes encouragés par cette récente activité et espérons que le mouvement derrière les compagnies de gestion des antimicrobiens au Canada continuera de prendre de l'essor.

\section{Conclusion}

La gestion des antimicrobiens peut être appuyée à l'échelle locale grâce à des campagnes d'éducation du public, comme «Des pilules contre tous les microbes? ». Les possibilités de lancement de campagnes de gestion des antimicrobiens au sein d'une collectivité sont innombrables, et aucune approche n'est plus juste qu'une autre. Le plus important, c'est de commencer.

\section{Remerciements}

Les auteurs tiennent à remercier Edith Blondel-Hill et Susan Fryters de leur travail précurseur pour l'établissement du programme «Des pilules contre tous les microbes? ».

\section{Conflit d'intérêts}

Aucun

\section{Financement}

Organisme de santé publique Alberta Health Services pour le financement en Alberta; Direction des services pharmaceutiques du ministère de la Santé de la Colombie-Britannique, pour le financement en ColombieBritannique.

\section{Références}

(1) Do Bugs Need Drugs? A Community Program for Wise Use of Antibiotics. http://www.dobugsneeddrugs.org/about/

(2) BC Centre for Disease Control. Antibiotic Utilization in the Province of British Columbia, 2013. Vancouver, BC: BC Centre for Disease Control; 2014 Nov. http://www.bccdc.ca/NR/rdonlyres/C3BFA092-7C55-46ED-BFC93CD91E593A5A/0/Reportonantibioticutilization2013_Nov12_FINAL.pdf

(3) World Health Organization (WHO). The evolving threat of antimicrobial resistance: Options for Action, 2012. Geneva: WHO; 2012. http://whqlibdoc.who.int/publications/2012/9789241503181_eng.pdf?ua=1

(4) Government of the United States. National Action Plan for Combating Antibiotic-resistant Bacteria. Washington: Government of the United States; 2015 Mar. https://www.whitehouse.gov/sites/default/files/docs/national_action_plan_for_combating_antiboticresistant_bacteria.pdf

(5) Government of Canada. Federal Action Plan on Antimicrobial Resistance and Use in Canada: Building on the Federal Framework for Action. 2015 Mar 31. Disponible en français: http://healthycanadians.gc.ca/alt/pdf/drugs-products-medicaments-produits/antibioticresistance-antibiotique/antimicrobial-framework-cadre-antimicrobiens-fra.pdf 